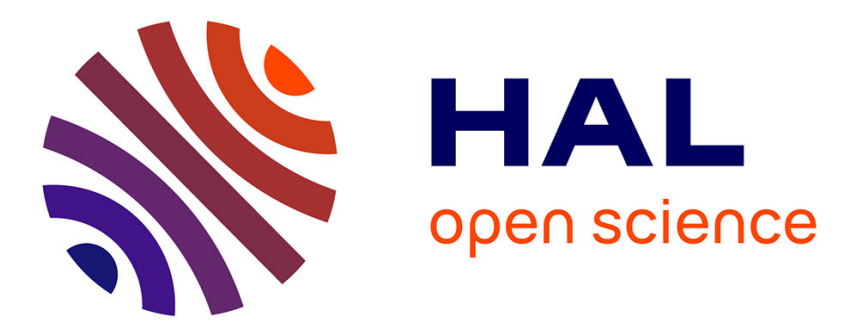

\title{
Etude de la capacité de stockage maxima dans un dispositif à transfert de charges en volume
}

\author{
D. Rigaud, D. Sodini, A. Touboul, K. Torbati
}

\section{To cite this version:}

D. Rigaud, D. Sodini, A. Touboul, K. Torbati. Etude de la capacité de stockage maxima dans un dispositif à transfert de charges en volume. Revue de Physique Appliquée, 1984, 19 (11), pp.945-950. 10.1051/rphysap:019840019011094500 . jpa-00245288

\section{HAL Id: jpa-00245288 https://hal.science/jpa-00245288}

Submitted on 1 Jan 1984

HAL is a multi-disciplinary open access archive for the deposit and dissemination of scientific research documents, whether they are published or not. The documents may come from teaching and research institutions in France or abroad, or from public or private research centers.
L'archive ouverte pluridisciplinaire HAL, est destinée au dépôt et à la diffusion de documents scientifiques de niveau recherche, publiés ou non, émanant des établissements d'enseignement et de recherche français ou étrangers, des laboratoires publics ou privés. 


\title{
Etude de la capacité de stockage maxima dans un dispositif à transfert de charges en volume $\left({ }^{1}\right)\left({ }^{*}\right)$
}

\author{
D. Rigaud, D. Sodini, A. Touboul et K. Torbati \\ Centre d'Electronique de Montpellier, Université des Sciences et Techniques du Languedoc, \\ 34060 Montpellier Cedex, France
}

(Reçu le 16 mai 1984, accepté le 17 août 1984)

\begin{abstract}
Résumé. - Pour minimiser les effets relatifs dus au courant d'osbcurité de génération recombinaison, au piégeage en volume et en surface et pour augmenter la sensibilité du système de lecture, un dispositif à transfert de charges (D.T.C.) doit pouvoir stocker et transférer une charge-signal importante. L'objet de ce travail est d'étudier la capacité de stockage en volume d'un D.T.C. à canal épitaxié en fonction des différents paramètres technologiques tant électriques que géométriques. Il sera de plus tenu compte des effets bi-dimensionnels dus à l'espace interélectrodes.
\end{abstract}

\begin{abstract}
A buried-channel C.C.D. must handle and transfer a large amount of signal charges to reduce parasitic effects related to dark current generation, bulk state trapping and to increase the sensitivity of the read-out circuitry. In this work, we study the charge handling capacity of an epi-layer B.C.C.D. as a function of the main technological and electrical parameters. Specific effects due to the gaps between the gate will be considered through a 2-D numerical simulation.
\end{abstract}

\section{Introduction.}

Un des paramètres caractéristiques d'un dispositif à transfert de charges (D.T.C.) à canal en volume est sa capacité à stocker une charge-signal importante tout en conservant une conduction en volume.

Cette charge-signal est définie pour un système de tension d'horloge donné et dépend de différents paramètres géométriques (épaisseur d'oxyde $d_{\mathrm{ox}}$ pour grille M.I.S., épaisseur $L$ de la couche épitaxiée, longueur $L_{\mathrm{g}}$ des grilles, espace interélectrodes $g .$. ) et électriques (dopage $N_{\mathrm{A}}$ du substrat et $N_{\mathrm{D}}$ de la couche épitaxiée, champ de claquage de l'oxyde, différence entre les amplitudes des signaux d'horloge...).

Comme cela a été montré pour une technologie à canal implanté [1], nous présentons ici une méthode rapide permettant de déterminer ce maximum de la charge-signal, méthode basée sur la résolution à une dimension de l'équation de Poisson. Ces calculs sont complétés par une simulation numérique à deux dimensions de la répartition des charges en équilibre et des potentiels sous les grilles de la structure D.T.C., à partir des valeurs numériques déterminées par le

( $\left.{ }^{1}\right)$ Ce travail a été effectué dans le cadre de l’opération « Modèles de composants » du G-CIS (CNRS). calcul 1-D. Cette simulation à deux dimensions sera faite pour différents types de technologies. Le schéma de principe du D.T.C. est donné sur la figure 1.

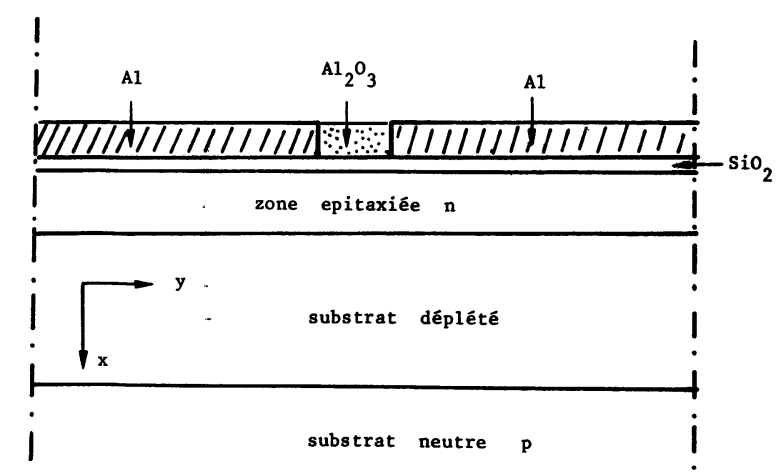

Fig. 1. - Coupe schématique d'une cellule de D.T.C. [Schematic cross-section of a B.C.C.D. cell.]

Les grilles peuvent être métalliques ou en silicium polycristallin, avec ou sans recouvrement, isolées par de l'alumine ou de la silice. Il est possible de passer d'une technologie à grille M.I.S. à une technologie à grille Schottky en faisant dans le modèle $d_{\mathrm{ox}}=0$. 
2. Etude de la capacité de stockage d'un D.T.C.

2.1 RéSolution analytique de l'équation de PoisSON À UNE DIMENSION [2]. - L'équation de Poisson

$$
\frac{\mathrm{d}^{2} V(x)}{\mathrm{d} x^{2}}=-\frac{\rho(x)}{\varepsilon_{\mathrm{sc}}}
$$

sera résolue compte tenu des approximations suivantes (cf. Fig. 2) :

i) la limite de la zone de déplétion côté $p$ est abrupte;

ii) les limites dans la région $\mathrm{n}$ de la charge-signal sont abruptes;

iii) l'oxyde n'est pas chargé.

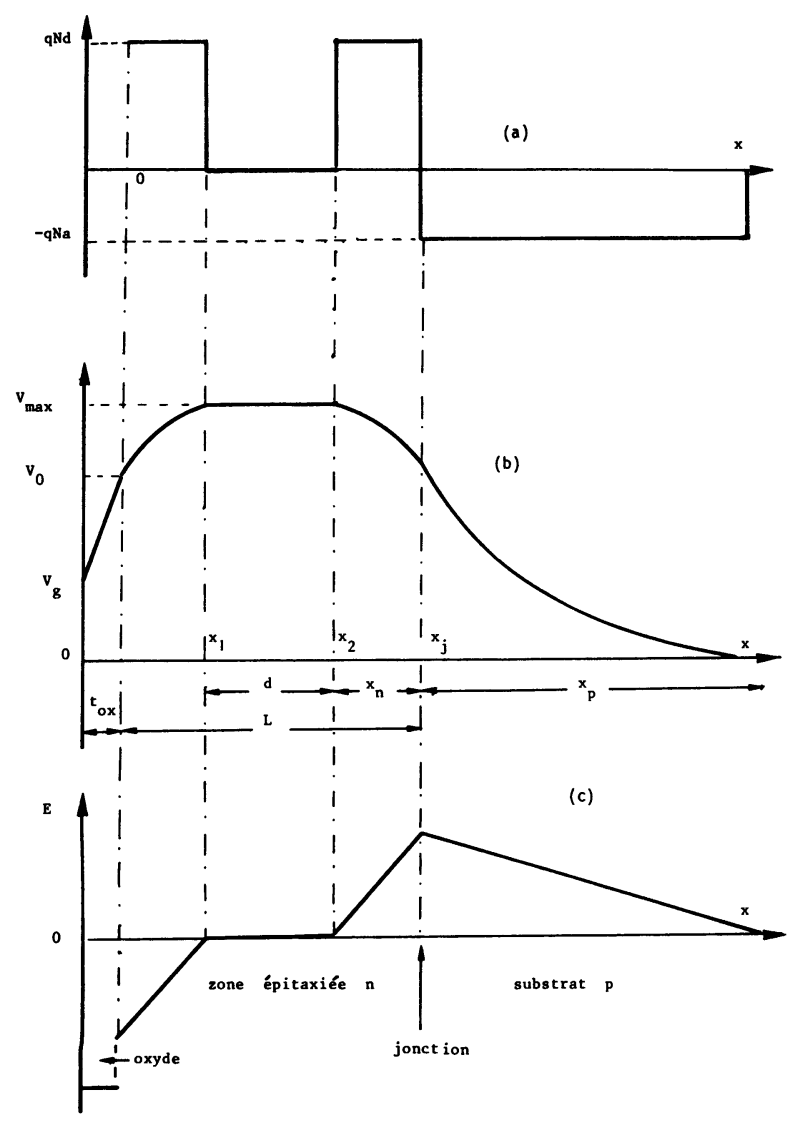

Fig. 2. - D.T.C. à grille M.I.S. : coupe sous une grille; a) densité de charge ; b) distribution du potentiel ; c) champ électrique.

[M.I.S. gate buried-channel C.C.D. : cross-section under one gate; a) assumed charge distribution; b) potential distribution; c) electric field profile.]

En intégrant l'équation de Poisson depuis l'oxyde jusqu'au maximum de potentiel dans le canal, on obtient :

$$
V_{\max }=V_{\mathrm{g}}+\frac{q N_{\mathrm{D}}}{\varepsilon_{\mathrm{ox}}} d_{\mathrm{ox}} x_{1}+\frac{q N_{\mathrm{D}}}{\varepsilon_{\mathrm{sc}}} x_{1}^{2}
$$

où $V_{\max }$ représente le maximum de potentiel dans le canal.

De même en intégrant l'équation de Poisson depuis le substrat, on obtient :

$$
V_{\max }=\frac{1}{2} \frac{q N_{\mathrm{D}}}{\varepsilon_{\mathrm{sc}}} x_{\mathrm{n}}^{2}+\frac{1}{2} \frac{q N_{\mathrm{A}}}{\varepsilon_{\mathrm{sc}}} x_{\mathrm{p}}^{2} .
$$

Compte tenu de la neutralité électrique de l'ensemble de la zone de charge d'espace de la jonction p-n $\left(N_{\mathrm{A}} x_{\mathrm{p}}=N_{\mathrm{D}} x_{\mathrm{n}}\right)$, l'équation (2) peut s'écrire :

$$
V_{\max }=\frac{1}{2} \frac{q N_{\mathrm{D}}}{\varepsilon_{\mathrm{sc}}} x_{\mathrm{n}}^{2}\left(1+\frac{N_{\mathrm{D}}}{N_{\mathrm{A}}}\right) \text {. }
$$

Soit $d$ l'épaisseur du canal neutralisé par la chargesignal $Q_{\mathrm{inj}}\left(Q_{\mathrm{inj}}=\right.$ charge/unité de surface).

On a

$$
d=\frac{Q_{\text {inj }}}{q N_{\mathrm{D}}} \quad \text { et } \quad x_{\mathrm{n}}=L-x_{1}-d .
$$

Les équations (3) et (4), compte tenu de (5), donnent :

$$
A x_{1}^{2}+B x_{1}+C=0 .
$$

Avec

$$
\begin{aligned}
& A=\frac{1}{2} \frac{q N_{\mathrm{D}}^{2}}{\varepsilon_{\mathrm{sc}} N_{\mathrm{A}}} \\
& B=-q N_{\mathrm{D}} \frac{d_{\mathrm{ox}}}{\varepsilon_{\mathrm{ox}}}+\frac{L-d}{\varepsilon_{\mathrm{sc}}}\left(1+\frac{N_{\mathrm{A}}}{N_{\mathrm{D}}}\right) \\
& C=-V_{\mathrm{g}}+\frac{1}{2} q \frac{N_{\mathrm{D}}}{\varepsilon_{\mathrm{sc}}}(L-d)^{2}\left(1+\frac{N_{\mathrm{A}}}{N_{\mathrm{D}}}\right) .
\end{aligned}
$$

La solution positive de (6) permet de calculer $V_{\max }$ et le champ électrique dans l'oxyde :

$$
E_{0}=\frac{q N_{\mathrm{D}}}{\varepsilon_{\mathrm{ox}}} x_{1} .
$$

2.2 INFLUENCE DES PARAMÈTRES GÉOMÉTRIQUES ET ÉLECTRIQUES. - Les courbes obtenues sont du type de celles données par Deyhimy [3] mais tiennent en plus compte de la présence d'une seconde grille portée à un potentiel différent : nous avons donc fait varier la différence des maxima de potentiels (cf. Fig. 3), créés par deux grilles l'une vide de charge-signal et polarisée à $V_{\mathrm{g} 1}=0 \mathrm{~V}$, l'autre polarisée à $V_{\mathrm{g} 2} \neq V_{\mathrm{g} 1}$ et contenant la charge-signal. La zone correspondant au fonctionnement correct du D.T.C. est limitée par les trois conditions suivantes :

Condition 1 : Le puits sous la grille chargée doit rester plus profond que celui sous la grille vide $(\Delta V>0)$.

Condition $2:$ Le champ à l'interface doit être inférieur à $10^{6} \mathrm{~V} / \mathrm{cm}$, valeur arbitraire, suffisamment forte pour interdire le claquage de l'oxyde [4] et 


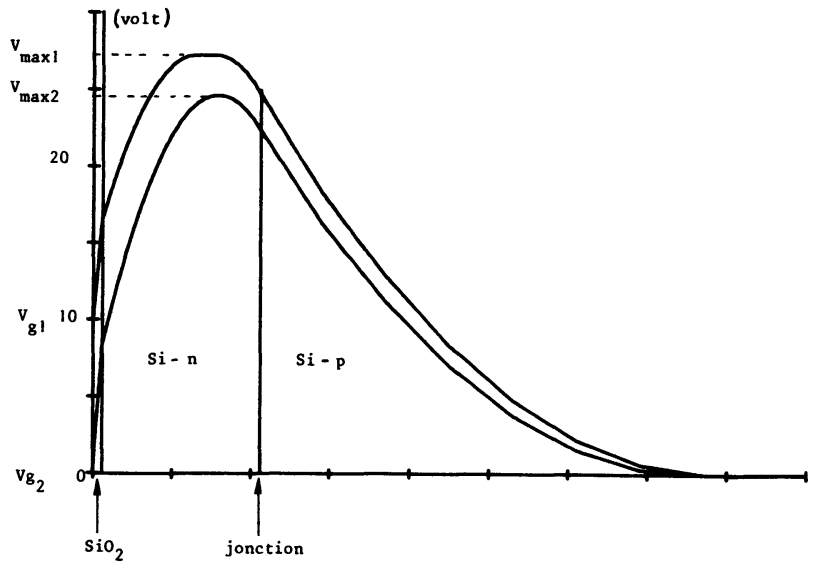

Fig. 3. - Distribution du potentiel sous deux grilles adjacentes.

[Potential distribution under two neighbouring gates.]

l'impact-ionisation. Cette valeur est néanmoins atteinte (Figs. 4 et 6) pour une charge-signal tendant vers zéro sous la grille de stockage.

Condition 3 : La différence de potentiel entre $V_{\max }$ correspondant au puits plein et $V_{0}$ le potentiel de l'interface doit être suffisamment grande pour éviter les interactions des porteurs avec la surface et assurer une conduction et un stockage en volume $\left(V_{\max }-V_{0}=V_{L}\right)$.

Sur la figure 4, nous avons représenté la variation de la différence $\left(V_{\max 2}-V_{\max 1}\right)$ en fonction de la charge-signal $Q_{\text {inj }}$ pour différentes valeurs du dopage $N_{\mathrm{D}}$ du canal. Cette étude a été faite pour deux types de système d'horloges : $0-10 \mathrm{~V}$ et $0-5 \mathrm{~V}$, sur des dispositifs ayant une épaisseur d'oxyde $d_{\text {ox }}$ de $0,12 \mu \mathrm{m}$, une épaisseur de canal $L$ de $2 \mu \mathrm{m}$ et un dopage du substrat $N_{\mathrm{A}}$ de $10^{15} \mathrm{at} / \mathrm{cm}^{3}$. On constate que le domaine de fonctionnement possible pour le D.T.C. se réduit considérablement lorsqu'on passe du système d'horloge $0-10 \mathrm{~V}$ à celui de $0-5 \mathrm{~V}$. Pour un substrat de $10^{15} \mathrm{at} / \mathrm{cm}^{3}$, le dopage du canal est compris entre $4 \times 10^{15}$ et $2 \times 10^{16} \mathrm{at} / \mathrm{cm}^{3}$. Par exemple, pour le système d'horloge 0-10 $\mathrm{V}$ une charge-signal de $5 \times 10^{-4} \mathrm{C} / \mathrm{m}^{2}$ dans un canal dopé à $10^{16} \mathrm{at} / \mathrm{cm}^{3}$ assure une différence de potentiel $V_{\max 1}-V_{\max 2}$ de $2 \mathrm{~V}$. Il faut constater que pour une charge-signal donnée, cette différence est d'autant plus grande que le dopage du canal est faible.

Sur la figure 5 , on a représenté les variations de $V_{\max 2}-V_{\max 1}$ en fonction de l'épaisseur $L$ du canal pour un système d'horloge $0-10 \mathrm{~V}$ et pour deux valeurs de la charge-signal $Q_{\mathrm{inj}}: 6 \times 10^{-4} \mathrm{C} / \mathrm{m}^{2}$ et $10^{-3} \mathrm{C} / \mathrm{m}^{2}$.

On constate ici que la différence sera d'autant plus grande pour une charge-signal donnée que l'épaisseur du canal sera plus petite et pour une épaisseur donnée que le dopage du canal sera faible.

La figure 6 représente les domaines possibles de fonctionnement lorsqu'on fait 'varier l'épaisseur d'oxyde de grille. La structure Schottky $\left(d_{\mathrm{ox}}=0\right)$ permet de stocker une charge-signal plus importante que celle associée à une grille M.I.S. d'épaisseur d'oxyde de $0,4 \mu \mathrm{m}$. La zone intermédiaire $\left(d_{\mathrm{ox}}=0,12 \mu \mathrm{m}\right)$ a déjà été représentée sur la figure 4 . Enfin, on a constaté que pour des dopages de substrat faibles $\left(N_{\mathrm{A}} \simeq 10^{14} \mathrm{at} / \mathrm{cm}^{3}\right)$, on augmentait la capacité de stockage. Ce résultat est en accord avec les valeurs communément utilisées pour $N_{\mathrm{A}}$ telles que $N_{\mathrm{A}} \simeq N_{\mathrm{D}} / 10$.

3. Etude de la distribution des potentiels et des charges dans la structure D.T.C. (Résolution numérique de l'équation de Poisson à 2 dimensions).

Nous avons résolu numériquement l'équation de Poisson à deux dimensions par une méthode de différences finies pour une structure test comprenant (cf. Fig. 1) deux demi-grilles de longueur $L_{\mathrm{g}} / 2=5 \mu \mathrm{m}$ séparées par un espace interélectrodes $g=1 \mu \mathrm{m}$.

Le programme permet d'avoir la carte des potentiels et la répartition des charges sous les grilles et dans l'espace interélectrodes. Sur les figures 7 et 8 , nous avons reporté, à titre indicatif, la distribution du potentiel sous une grille polarisée à $10 \mathrm{~V}$, avec charges ainsi que leur répartition.

La figure 7 montre la carte des potentiels dans la structure test polarisée par un système d'horloge $0-10 \mathrm{~V}$ et contenant une charge de $6 \times 10^{-4} \mathrm{C} / \mathrm{m}^{2}$ (soit $3 \times 10^{-9} \mathrm{C} / \mathrm{m}$ pour une demi-grille de longueur $\left.L_{\mathrm{g}} / 2=5 \mu \mathrm{m}\right)$. A l'interface oxyde-semiconducteur on remarque un extremum de potentiel $(>20 \mathrm{~V})$ situé au milieu de l'espace interélectrodes. Ce puits de potentiel s'atténue pour disparaître complètement au voisinage de la ligne des maxima de potentiel.

La répartition des charges (donnée Fig. 8), montre que le paquet de charges occupe complètement l'espace interélectrodes et déborde sous $G_{1}$. La quantité de charges $\left(3 \times 10^{-9} \mathrm{C} / \mathrm{m}\right)$ correspondant au point $A$ de l'abaque représentée figure 4 est limite pour ce type de fonctionnement.

Pour une charge plus faible (correspondant au point B de l'abaque figure 4), dont la répartition est donnée sur la figure 9, on constate que le stockage des charges est tel que les porteurs sont mieux localisés sous $G_{2}$.

Ces résultats numériques confirment la validité et l'intérêt des calculs analytiques simples présentés plus haut.

\section{Conclusion.}

Nous avons montré que, par un modèle simple mettant en jeu la résolution analytique de l'équation de Poisson à 1 dimension, il était possible de définir des critères technologiques pour la conception de D.T.C. silicium correspondant à un domaine possible de fonctionnement. L'influence des différents paramètres 


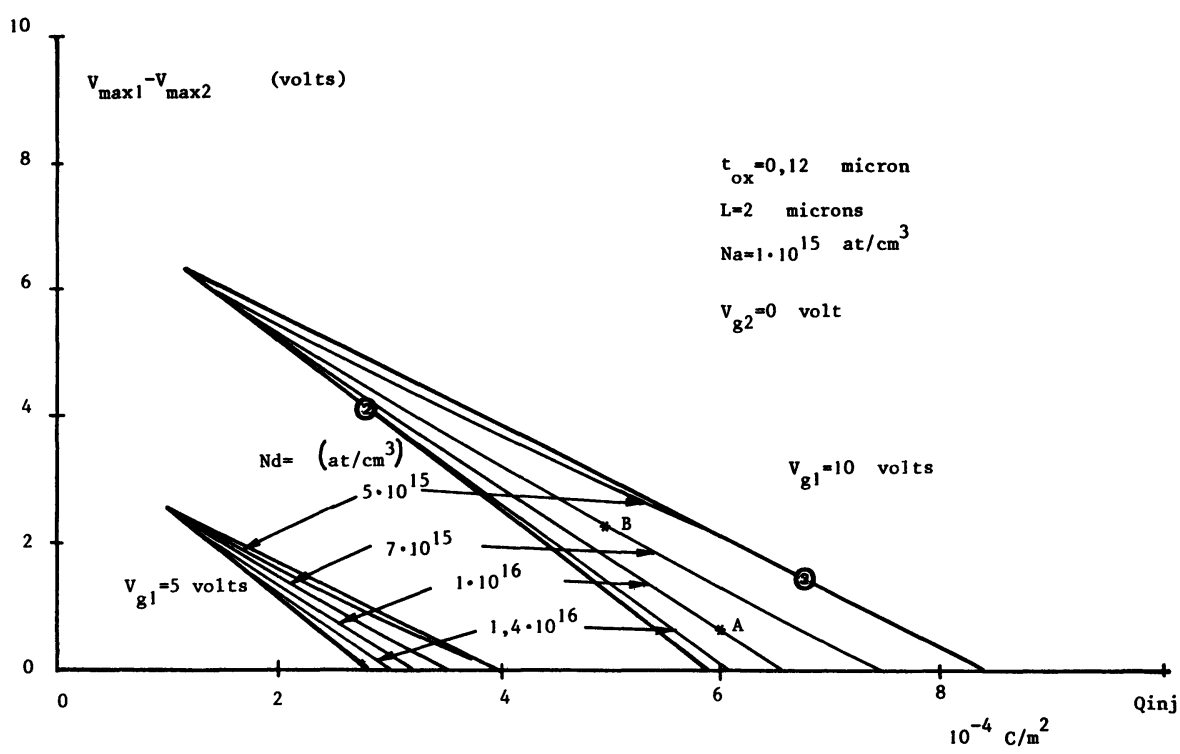

Fig. 4. - Différence des potentiels maximum de deux puits voisins calculée en fonction de la charge injectée et du dopage de la zone épitaxiée et ce pour deux amplitudes d'horloges $(5 \mathrm{~V}$ et $10 \mathrm{~V})$. (2) et (3) sont les limites du domaine de fonctionnement dues aux conditions 2 et $3 \mathrm{du}$ texte (cf. $§ 2.2$ ).

[Calculated difference maximum voltage as a function of stored charge density and epitaxial doping values for two clock swings $(5 \mathrm{~V}$ and $10 \mathrm{~V})$. (2) and (3) refer to the conditions two and three (see Sect. 2.2).]

Fig. 5. - Différence des potentiels maximum de deux puits voisins calculée en fonction de l'épaisseur et du dopage de la zone épitaxiée et ce pour deux valeurs de la charge-signal.

[Calculated difference maximum voltage as a function of the channel thickness and epitaxial doping values for two charge-packets.]

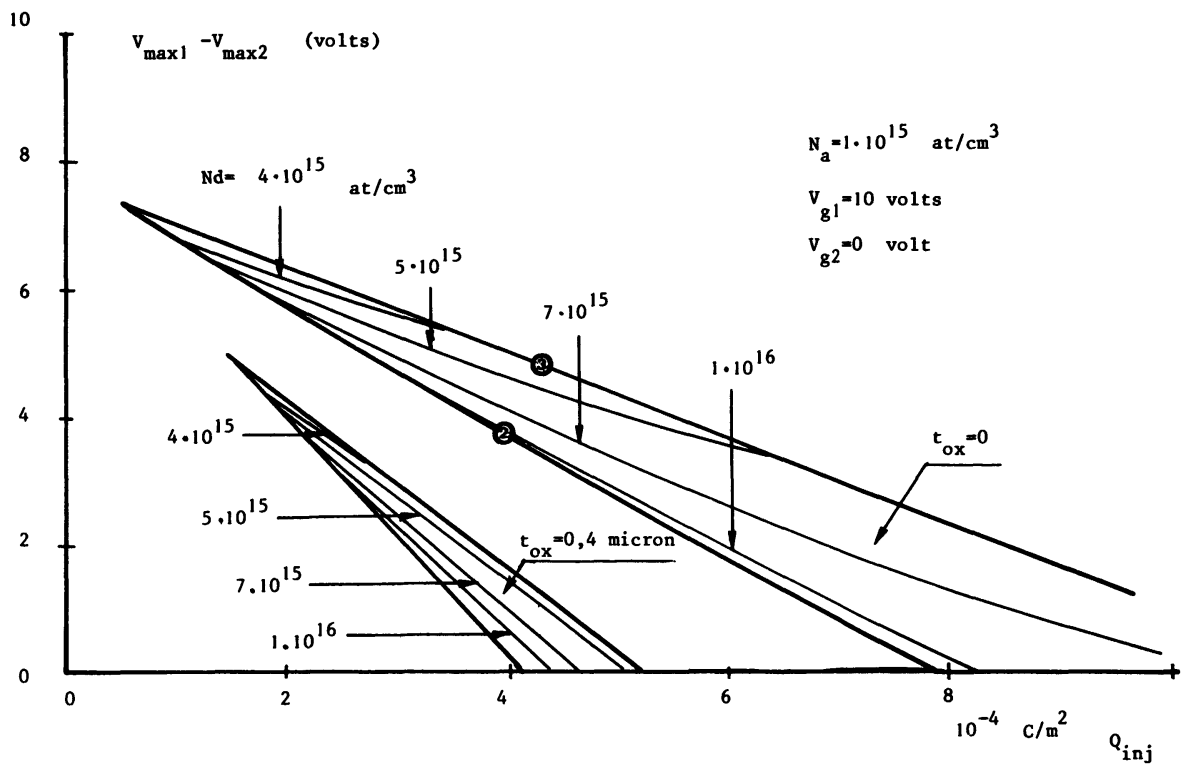

Fig. 6. - Différence des potentiels maximum de deux puits voisins calculée en fonction de la charge injectée et du dopage de la zone épitaxiée et ce pour deux valeurs d'épaisseur d'oxyde.

[Calculated difference maximum voltage as a function of stored charge density and epitaxial doping values for two oxide thicknesses.] 

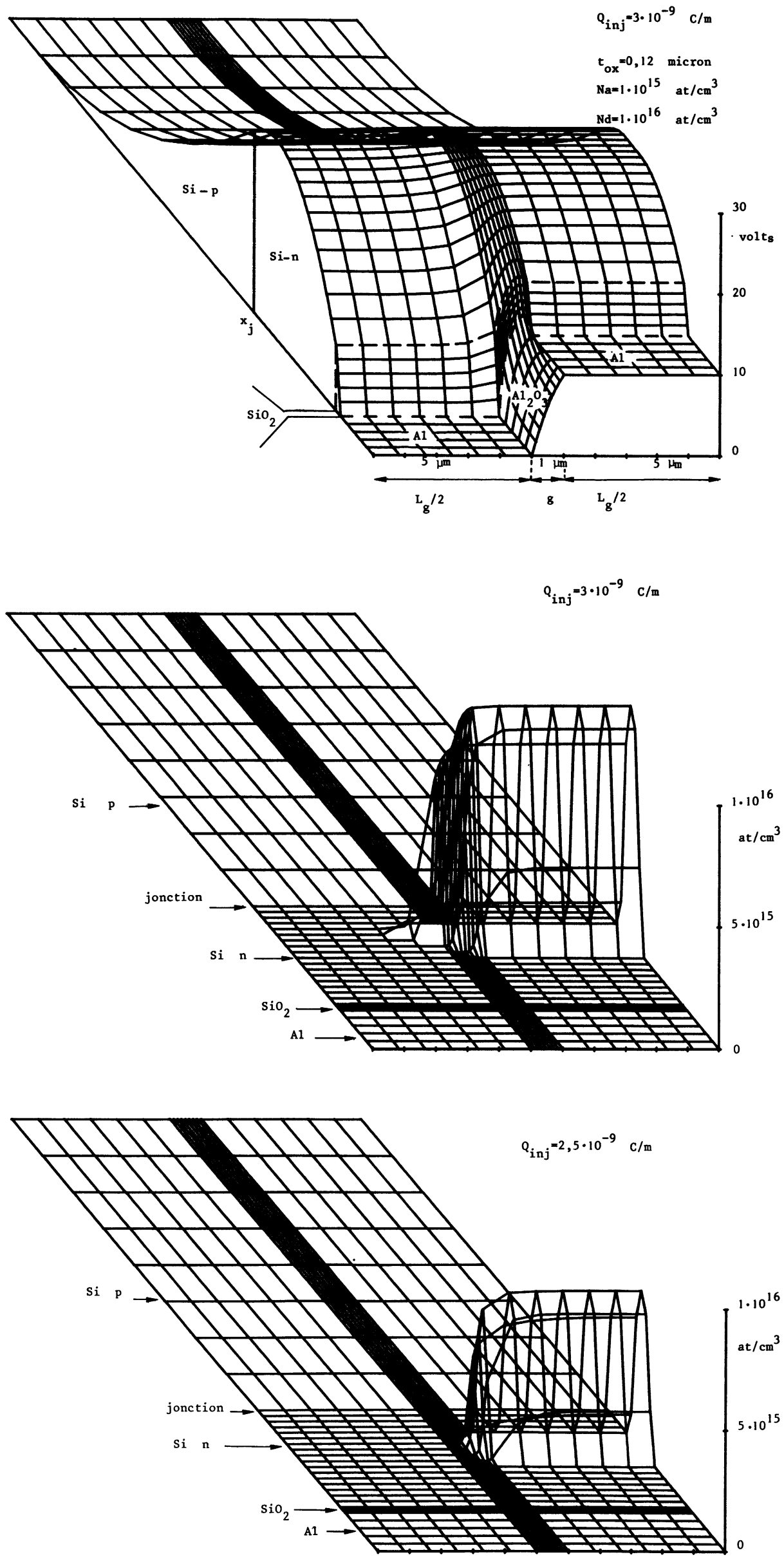

REVUE DE PHYSIQUE APPLIQUÉE. - T. 19, № 11, NOVEMBRE 1984
Fig. 7. - Distribution du potentiel sous les deux grilles de la cellule élémentaire de transfert.

[Potential distribution under the gates of the transfer elementary cell.]

Fig. 8. - Répartition de la densité de charge correspondant au point $A$ de la figure 4.

[Charge density distribution corresponding to the point $\mathbf{A}$ of figure 4.]

Fig. 9. - Répartition de la densité de charge correspondant au point $B$ de la figure 4 .

[Charge density distribution corresponding to the point $B$ of figure 4.] 
électriques et géométriques a été mise en évidence. Il apparaît clairement que, contrairement à ce que l'on pouvait penser, la meilleure capacité de stockage des charges-signal est obtenue lorsqu'on diminue à la fois le dopage du canal et son épaisseur.

La validité de ce modèle a été confirmée en résolvant numériquement l'équation de Poisson à deux dimen- sions pour obtenir les cartes de potentiels et les distributions de charges dans le D.T.C.

Nous avons mis en évidence le débordement des charges-signal sous la grille voisine à celle effectuant le stockage lorsque la conception de la structure correspond à la limite des abaques obtenues par le modèle à une dimension.

\section{Bibliographie}

[1] Chatterjee, P. K., Taylor, G. W., « Optimum Scaling of Buried-channel CCD's ", I.E.E.E. Trans. Electron Devices ED-27, $\mathrm{n}^{\circ} 3$ (1980) 553-562.

[2] HAKen, R. A., "A comparison of static bulk channel charge-coupled device characteristics using uniform, Gaussian and measured impurity distributions ", Solid State Electron. 20 (1977) 789-797.

[3] Deyhimy, I., Eden, R. C., " GaAs and related heterojunction charge-coupled devices », I.E.E.E. Trans. Electron Devices ED-27, no 6 (1980) 1172-1180.

[4] SzE, S. M., "Physics of semiconductor devices " (J. Willey and Sons, Editors). 